\title{
Mortality and Neurological Outcomes in Out-of-Hospital Cardiac Arrest Patients With and Without Targeted Temperature Management in a Multiethnic Asian Population
}

Wan Jing Tay, ${ }^{1}$ MBBS, Huihua Li $\underline{1},{ }^{2}$ PhD, Andrew FW $\underline{\text { Ho }},{ }^{3,4,5}$ MBBS, MMed, Ching Hui Sia, ${ }^{6,7}{ }_{M B B S, ~ M R C P}$ (UK), MMed (Int Med), Georgina GJ Kwek, ${ }^{1}$ MBBS, Sohil Pothiawala, ${ }^{1}$ MBBS, MRCSEd (A\&E), Nur Shahidah, ${ }^{1}$ BA (Psych with Business), Kenneth BK $\underline{\text { Tan }},{ }^{1}$ MBBS, MCEM, Aaron SL Wong, ${ }^{8}$ MBBChir MRCP (UK), Duu Wen Sewa, ${ }^{9}$ MBBS, MRCP (UK), Eric TS Lim, ${ }^{8}$ MBBChir, MRCP, Chee Tang $\underline{\text { Chin }},{ }^{8}$ MB ChB (Hons), MRCP (UK), Marcus EH Ong, ${ }^{1,10}$ MBBS, FRCSEd (A\&E)

\begin{abstract}
Introduction: The use of targeted temperature management (TTM) is increasing although adoption is still variable. We describe our 6-year experience and compare the mortality and neurological outcomes of out-of-hospital cardiac arrest (OHCA) patients with and without the use of TTM in a multiethnic Asian population. Materials and Methods: We performed a retrospective observational study at a tertiary academic medical centre. $\mathrm{OHCA}$ survivors admitted to our hospital between April 2010-December 2016 were included. Outcomes of interest were 30-day mortality postresuscitation, Cerebral Performance Category (CPC) and Overall Performance Category (OPC) scores. Results: A total of 121 of 261 patients $(46.3 \%)$ underwent TTM. TTM patients were younger (TTM 60.0 years old vs no TTM 63.7 years old, $P=0.047$ ). There was no difference in the initial arrest rhythm of shockable origin between the 2 groups $(P=0.289)$. There was suggestion of lower 30-day mortality (TTM $24.3 \%$ vs no TTM $31.4 \%, P=0.214$ ), higher and good CPC/OPC scores (TTM $19.0 \%$ vs no TTM $15.7 \%, P=0.514$ ) with TTM although this did not reach statistical significance. On multivariable logistic regression analysis, TTM was not associated with 30-day mortality $(P=0.07)$. However, older age, initial non-shockable rhythm and increased duration from arrest to return of spontaneous circulation were associated with increased mortality. Malay ethnicity was associated with a poorer CPC/ OPC score. Conclusion: Adoption and outcomes of TTM postresuscitation is variable and there is still a need to optimise management of the identified predictors of survival and good neurological outcomes while TTM is being used.
\end{abstract}

Ann Acad Med Singapore 2020;49:127-36

Key words: Heart attack, Neurological function, Neuroprotection, Therapeutic hypothermia

\section{Introduction}

Targeted temperature management (TTM) is an established but evolving modality for treating out-ofhospital cardiac arrest (OHCA) patients. There have been significant advances in the management of OHCA patients and prevention of sudden cardiac death, but much is still unknown. ${ }^{1,2}$ Two landmark clinical studies performed in Europe and Australia demonstrated that the initiation of TTM improved mortality and neurological outcomes. ${ }^{3,4}$
Since then, the International Liaison Committee on Resuscitation and the American Heart Association have recommended the use of TTM after cardiac arrest as soon as possible after return of spontaneous circulation (ROSC). ${ }^{5}$ They further released an advisory statement regarding TTM in 2015 recommending it for adults with OHCA who have an initial shockable or non-shockable rhythm but remain unresponsive after ROSC and TTM for at least 24 hours. ${ }^{6}$

${ }^{1}$ Department of Emergency Medicine, Singapore General Hospital, Singapore

${ }^{2}$ Division of Medicine, Singapore General Hospital, Singapore

${ }^{3}$ SingHealth Duke-National University of Singapore Emergency Medicine Academic Clinical Programme, Singapore

${ }^{4}$ National Heart Research Institute Singapore, National Heart Centre, Singapore

${ }^{5}$ Cardiovascular and Metabolic Disorders Programme, Duke-National University of Singapore Medical School, Singapore

${ }^{6}$ Department of Cardiology, National University Heart Centre, Singapore

${ }^{7}$ Department of Medicine, Yong Loo Lin School of Medicine, National University of Singapore

${ }^{8}$ Department of Cardiology, National Heart Centre, Singapore

${ }^{9}$ Department of Respiratory and Critical Care Medicine, Singapore General Hospital, Singapore

${ }^{10}$ Health Services and Systems Research, Duke-National University of Singapore Medical School, Singapore

Address for Correspondence: Dr Sia Ching Hui, Department of Cardiology, National University Heart Centre, Singapore, 1E Kent Ridge Road, Level 9 ,

NUHS Tower Block, Singapore 119228.

Email: siachinghui@gmail.com 
Almost 2 decades on and despite the recommendations, there are still areas of controversy. In many centres, adoption is still based on individual clinician and institutional discretion. There are unresolved issues of optimal targeted temperature, haemodynamic and neurological monitoring methods, and the treatment of fever among various other issues. ${ }^{7}$ Guidelines were developed based on studies of Caucasian populations, and the landmark studies recruited only patients with OHCA and ventricular fibrillation. In Asia, experience with the use of TTM is still developing and is variable. ${ }^{8,9}$ Most of the larger published Asian studies involved countries with ethnically homogenous populations (The Korea Hypothermia Network Registry from Korea ${ }^{10}$ and the J-PULSE-HYPO study registry from Japan). ${ }^{11}$ In other parts of Asia, there are single-centre studies from Thailand $\mathrm{d}^{12,13}$ and Taiwan, ${ }^{14}$ which again consist of ethnically homogenous populations. A pilot study from our own group in Singapore ${ }^{15}$ in 2014 showed that a therapeutic hypothermia protocol could be safely implemented, but consisted of a small sample size of TTM patients $(n=25)$. Furthermore, ethnic differences have also been shown to affect neurological outcomes in studies performed in the United States of America (USA). In particular, the nonWhite ethnic groups were demonstrated to have poorer neurological outcomes after cardiac arrest. ${ }^{16,17}$ Alocal study of a paediatric population with in-hospital cardiac arrests did not show any ethnic differences, although the sample size was small. ${ }^{18}$ Whether ethnicity is a factor affecting neurological outcomes in OHCA in our local context is not known.

As such, the primary aim of this study was to describe the use of TTM on OHCA patients admitted to a tertiary academic medical centre serving a multiethnic adult Asian population over a 6 -year period. The outcomes of interest were 30-day mortality, good cerebral performance category (CPC) and overall performance category (OPC) scores. We aimed to identify predictors of improved 30-day mortality and $\mathrm{CPC} / \mathrm{OPC}$ scores.

\section{Materials and Methods}

This was a retrospective observational study using Singapore data from the Pan-Asian Resuscitation Outcomes Study (PAROS) ${ }^{19}$ and our institution's electronic medical records. PAROS is a collaborative research network that collects data prospectively and aims to improve outcomes of prehospital and emergency care in the Asia-Pacific region.

Data was collected on all OHCA patients who presented to the Singapore General Hospital Emergency Department and survived to admission between April 2010-December 2016. The inclusion criteria for TTM were as follows (although discretion was given to the attending clinician as to whether to eventually initiate TTM): 1) OHCA patients with all-presenting rhythms and sustained ROSC after cardiac arrest for $>30$ minutes, 2) patients who were comatose (with a Glasgow Coma Score of $\leq 8$ ) or who were unresponsive postresuscitation, and 3) patients who were haemodynamically stable with systolic blood pressure (BP) $>90 \mathrm{mmHg}$ (with or without inotropic support). The exclusion criteria included OHCA patients deemed unsuitable for further active intervention, those who regained consciousness, patients with persistent hypotension despite fluid and/or vasopressor support, those with a positive pregnancy test in females $<50$ years old and trauma patients. Patient characteristics collected include demographics (age, gender, race and comorbidities), arrest details (location, time, witness, bystander cardiopulmonary resuscitation [CPR]), first presenting rhythm, time of ROSC, aetiology (cardiac vs non-cardiac) and post-ROSC interventions (percutaneous coronary intervention [PCI], coronary artery bypass graft, implantable cardioverter-defibrillator use). Prehospital data was obtained from the PAROS registry. The time of arrest was estimated and based on the time the patient was found unresponsive/unconscious with no pulse and no/abnormal breathing by relatives or a bystander. The time of ROSC was taken as the first ROSC confirmed by a palpable pulse and electrocardiogram in the emergency department. The main primary outcomes measured at 30 days postresuscitation were mortality, discharge status, $\mathrm{CPC}$ and OPC scores. ${ }^{20} \mathrm{~A}$ good CPC score was defined as having a score of 1 or 2 according to the Glasgow-Pittsburg Outcome Categories. Neurological status was assessed by attending physician either upon discharge or at the 30 days postarrest. Mortality was assessed within 30 days of cardiac arrest. This would be documented in the inpatient discharge summary notes and extracted accordingly in the case record form by a clinical research coordinator.

Patients received standard intensive care as per the treating physician. Sedation was started with intravenous midazolam ( $0.02-0.08 \mathrm{mg} / \mathrm{kg}$ over $1-2$ minutes, initially). Doses were uptitrated to achieve sedation as BP tolerated. Paralysis was induced with atracurium $(0.4-0.5 \mathrm{mg} / \mathrm{kg}$ intravenous bolus, initially) and titrated as required. The target temperature was $34^{\circ} \mathrm{C}$. The emergency department would initiate cooling with cold saline infusion and surface icepacks until the cooling device was commenced in the intensive care unit. Ice packs were subsequently replaced by a wearable cooling suit in July 2015 as standard-of-care treatment for eligible patients. ${ }^{21}$ Core temperature was monitored via rectal or oesophageal temperature probe. The target temperature of $34^{\circ} \mathrm{C}$ was maintained for 24 hours followed by passive rewarming to $36.5^{\circ} \mathrm{C}$ by setting the cooling unit accordingly. The aim was to increase temperature by $1^{\circ} \mathrm{C}$ every 4 hours and achieve rewarming over at least 12 hours. The patient would be warmed no 
faster than $1{ }^{\circ} \mathrm{C}$ in 4 hours over a period of 8 hours. A detailed protocol can be found in the Appendix.

Data management was carried out using the REDCap data entry platform. ${ }^{22}$ All data analyses were performed using SPSS version 23.0 (IBM Corp., Armonk, New York, USA). For categorical data, frequency tables, together with $95 \%$ confidence interval(CI) were reported. Continuous variables were reported using mean, and where appropriate the $95 \%$ $\mathrm{CI}$ and median with interquartile range were reported. Those with $P$ values $<0.2$ were included for consideration to develop a multivariable logistic regression model. The significance level was set at 0.05 .

The study protocol was approved by SingHealth Centralised Institutional Review Board with waiver of informed consent.

\section{Results}

There were 1171 OHCA patients who presented to our emergency department from April 2010-December 2016, and 262 survived to admission (Fig. 1). Of the 262, 1 was excluded due to incomplete documentation of relevant TTM variables. Out of 261 that were included in this study, 121 (46.4\%) underwent TTM and 140 (53.6\%) either did not undergo TTM or were ineligible for TTM.

Table 1 shows the baseline characteristics of OHCA patients, grouped by whether they underwent TTM. The mean age of those who underwent TTM was about 3 years younger than those who had not (60.0 years old vs 63.7 years old, $P=0.047$ ). Significant differences between these 2 groups were age, race, incidence of stroke, year of arrest, presence of witness to the arrest and the person who performed initial CPR. Among patients who did not undergo TTM, survival to discharge was $24.3 \%$ - compared to $31.4 \%$ in those who underwent TTM - although not statistically significant $(P=0.200)$. There was also no significant difference in good neurological outcome (CPC 1-2) between patients who underwent TTM and those without (19.0\% vs $15.7 \%, P=0.514)$. The 6 -year trend of TTM use in our population is shown in Figure 2.

In the multivariable logistic regression analysis (Table $2)$, TTM was not associated with 30 -day mortality $(P=$ $0.07)$. However, 3 variables were found to be significantly associated. These were increased age (odds ratio [OR] 1.04, 95\% CI 1.02-1.07), longer time between arrest and first ROSC (OR 1.06, 95\% CI 1.04-1.09) and an initial nonshockable arrest rhythm (OR 6.62, 95\% CI 3.16-14.54). Positive predictor of good neurological outcome was presence of prehospital defibrillation (OR 3.82, 95\% CI 1.28-12.53). Negative predictors for good neurological outcome were age, duration between time of arrest and first ROSC, Malay ethnicity (with Chinese as the reference group, adjusted OR $0.10,95 \%$ CI $0.01-0.65, P=0.0319$ ) and first CPR initiated by emergency medical services (EMS)/private ambulance (Table 3 ).

\section{Discussion}

The key findings from our paper are as follows: 1) patients who underwent TTM were younger and fewer had a past history of stroke, 2) while there was a suggestion of improved survival to discharge and better CPC/OPC scores in the TTM group, this did not reach statistical

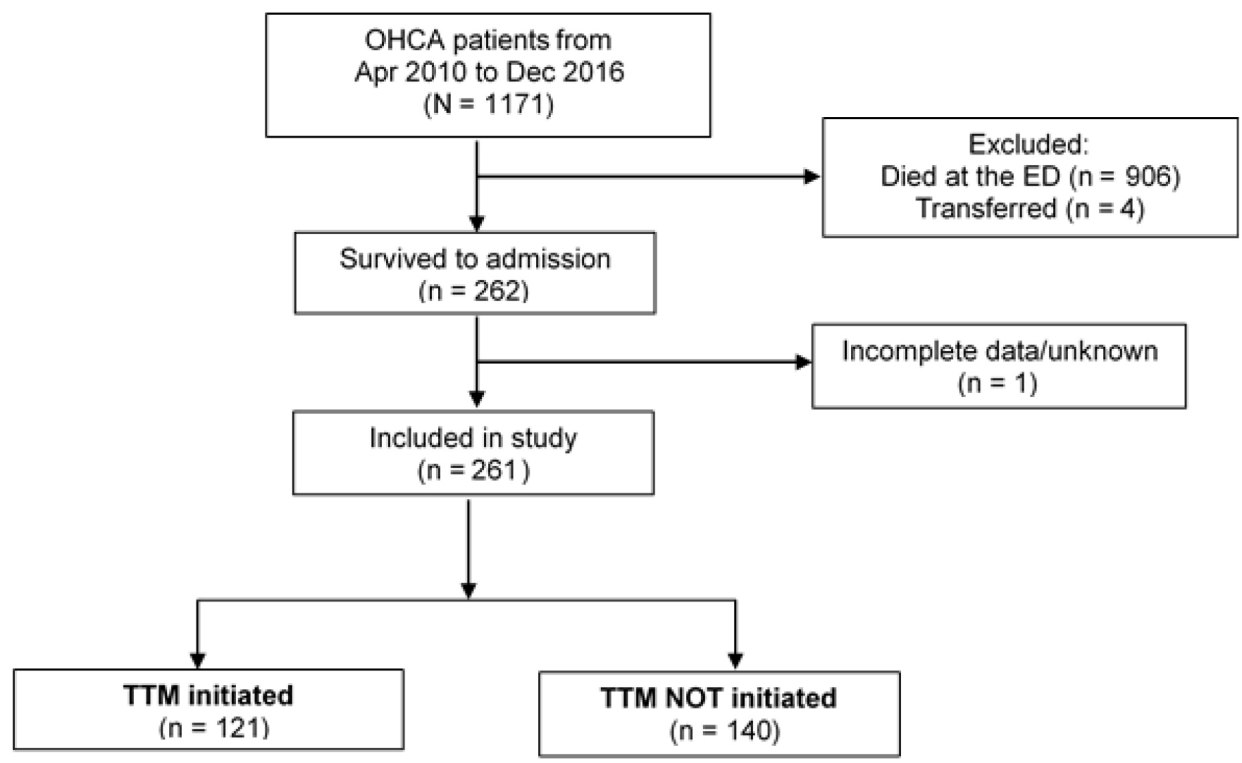

Fig. 1. Flow chart of out-of-hospital cardiac arrest patients included in the study. ED: Emergency department; OHCA: Out-of-hospital cardiac arrest; TTM: Targeted temperature management 
Table 1. Baseline Characteristics of Out-of-Hospital Cardiac Arrest Patients With and Without Targeted Temperature Management

\begin{tabular}{|c|c|c|c|}
\hline Characteristic, n (\%) & $\operatorname{TTM}(n=121)$ & Non-TTM $(n=140)$ & $P$ Value \\
\hline Mean age in years (IQR) & $60.0(48-72)$ & $63.7(55-76)$ & 0.047 \\
\hline Race & & & 0.009 \\
\hline Malay & $12(9.9)$ & $12(8.6)$ & \\
\hline Indian & $11(9.1)$ & $16(11.4)$ & \\
\hline Others & $4(3.3)$ & $23(16.4)$ & \\
\hline Gender, male & $92(76.0)$ & $96(68.6)$ & 0.214 \\
\hline \multicolumn{4}{|l|}{ Premorbidities } \\
\hline No medical history & $24(19.8)$ & $19(13.6)$ & 0.185 \\
\hline Unknown medical history & $4(3.3)$ & $7(5.0)$ & 0.552 \\
\hline Heart disease & $45(37.2)$ & $62(44.3)$ & 0.258 \\
\hline Diabetes & $28(23.1)$ & $42(30.0)$ & 0.262 \\
\hline Cancer & $8(6.6)$ & $11(7.9)$ & 0.813 \\
\hline Hypertension & $61(50.4)$ & $66(47.1)$ & 0.621 \\
\hline Renal disease & $13(10.7)$ & $20(14.3)$ & 0.457 \\
\hline Respiratory disease & $24(19.8)$ & $21(15.0)$ & 0.327 \\
\hline Hyperlipidaemia & $42(34.7)$ & $50(35.7)$ & 0.897 \\
\hline Stroke & $7(5.8)$ & $19(13.6)$ & 0.040 \\
\hline Mean estimated time in minutes between cardiac arrest and first sustained ROSC (IQR) & $48(32-64)$ & $46(31-61)$ & 0.656 \\
\hline Year of incidence & & & 0.002 \\
\hline 2010 & $5(4.1)$ & $20(14.3)$ & \\
\hline 2011 & $13(10.7)$ & $24(17.1)$ & \\
\hline 2012 & $12(9.9)$ & $23(16.4)$ & \\
\hline 2014 & $32(26.4)$ & $21(15.0)$ & \\
\hline 2015 & $15(12.4)$ & $19(13.6)$ & \\
\hline 2016 & $19(24.0)$ & $17(12.1)$ & \\
\hline Arrest witnessed by & & & 0.001 \\
\hline Not witnessed & $17(14.0)$ & $43(30.7)$ & \\
\hline Emergency medical services/private ambulance & $12(9.9)$ & $20(14.3)$ & \\
\hline Bystander & $92(76.0)$ & $77(55.0)$ & \\
\hline Initial cardiopulmonary resuscitation performed by & & & 0.029 \\
\hline Not performed & $4(3.3)$ & $8(5.7)$ & \\
\hline Emergency medical services/private ambulance & $54(44.6)$ & $79(56.4)$ & \\
\hline Bystander & $59(48.8)$ & $53(37.9)$ & \\
\hline Hospital staff & $4(3.3)$ & $0(0)$ & \\
\hline Cardiac cause of arrest & $85(70.2)$ & $85(60.7)$ & 0.063 \\
\hline Initial rhythm & & & 0.289 \\
\hline Shockable & $34(28.1)$ & $48(34.3)$ & \\
\hline Non-shockable & $87(71.9)$ & $92(65.7)$ & \\
\hline
\end{tabular}

IQR: Interquartile range; ROSC: Return of spontaneous circulation; TTM: Targeted temperature management 
Table 1. Baseline Characteristics of Out-of-Hospital Cardiac Arrest Patients With and Without Targeted Temperature Management (Cont'd)

\begin{tabular}{|c|c|c|c|}
\hline Characteristic, n (\%) & $\operatorname{TTM}(\mathrm{n}=121)$ & Non-TTM $(n=140)$ & $P$ Value \\
\hline Prehospital defibrillation & $52(43.0)$ & $54(38.6)$ & 0.528 \\
\hline Emergency percutaneous coronary intervention/stent performed & $34(28.1)$ & $29(20.7)$ & 0.192 \\
\hline Coronary artery bypass grafting performed & $1(0.8 \%)$ & $0(0)$ & 0.464 \\
\hline \multicolumn{4}{|l|}{ Outcomes } \\
\hline Survived to discharge & $38(31.4)$ & $34(24.3)$ & 0.214 \\
\hline Good Cerebral Performance Category score $(1-2)$ & $23(19.0)$ & $22(15.7)$ & 0.514 \\
\hline
\end{tabular}

IQR: Interquartile range; ROSC: Return of spontaneous circulation; TTM: Targeted temperature management

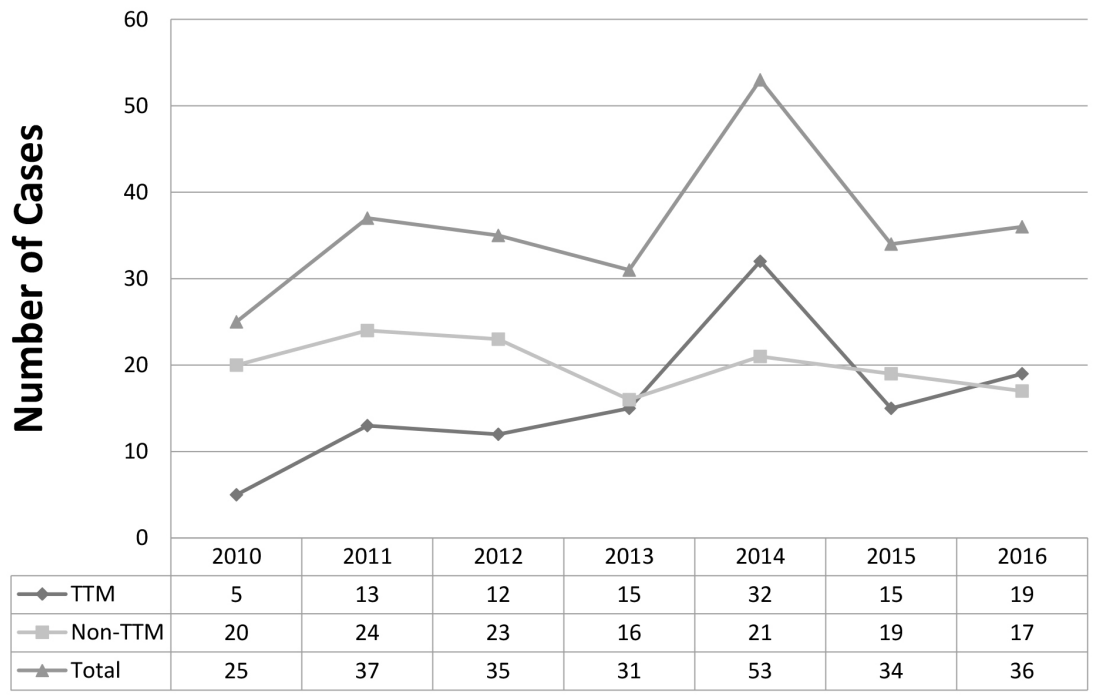

Fig. 2. Trend of targeted temperature management (TTM) over the 6 years of the study.

Table 2. Multivariable Analysis of Predictors of Mortality

\begin{tabular}{|c|c|c|c|c|}
\hline Variable & Number of Survivals & Number of Deaths & Odds Ratio (95\% CI) & $P$ Value \\
\hline Mean age & 61 & 178 & $1.04(1.02-1.07)$ & 0.0005 \\
\hline Time between arrest and ROSC (downtime) & 61 & 178 & $1.06(1.04-1.09)$ & $<0.0001$ \\
\hline \multicolumn{5}{|l|}{ First arrest rhythm } \\
\hline Shockable & 37 & 39 & Reference & \\
\hline Non-shockable & 24 & 139 & $6.62(3.16-14.54)$ & $<0.0001$ \\
\hline
\end{tabular}

CI: Confidence interval; ROSC: Return of spontaneous circulation

significance, 3) an older age, a longer time from cardiac arrest to ROSC and presence of a non-shockable initial rhythm were predictors of mortality after adjustment, 4) a positive predictor of good neurological outcome was the use of prehospital defibrillation, and 5) Malay ethnicity as compared to Chinese ethnicity as the reference was associated with a poorer neurological outcome.

We compared the characteristics between our study population and previously published TTM populations in developed Asia (South Korea and Japan), a centre in developing Asia and a registry based in the USA. ${ }^{10,11,13,23}$ The age across the populations was similar (about 60 years old) and there was a male predominance. Our TTM population had a higher proportion of patients who had witnessed arrest, but they were less likely to have an initial documented shockable rhythm. The difference in practices observed may be due to geographical size, provision of EMS services and individual hospital practices and would be of 
Table 3. Multivariable Analysis of Neurological Outcome (CPC)

\begin{tabular}{|c|c|c|c|c|}
\hline Variable & Poor CPC Score (3 - 4) & Good CPC Score $(1-2)$ & Odds Ratio $(95 \%$ CI) & $P$ Value \\
\hline Mean age & 216 & 45 & $0.95(0.92-0.98)$ & 0.0049 \\
\hline Time between arrest and ROSC (downtime) & 203 & 36 & $0.90(0.86-0.93)$ & $<0.0001$ \\
\hline \multicolumn{5}{|l|}{ Race } \\
\hline Chinese & 150 & 30 & Reference & \\
\hline Malay & 21 & 3 & $0.10(0.01-0.65)$ & 0.0319 \\
\hline Indian & 24 & 3 & - & 0.9924 \\
\hline Others & 21 & 9 & $1.89(0.50-7.24)$ & 0.3460 \\
\hline \multicolumn{5}{|l|}{ Who initiated CPR } \\
\hline No CPR initiated & 6 & 6 & Reference & \\
\hline Emergency medical services/private ambulance & 120 & 13 & $0.07(0.01-0.66)$ & 0.0237 \\
\hline Bystander & 87 & 25 & $0.19(0.01-2.00)$ & 0.1762 \\
\hline Hospital staff & 3 & 1 & $0.13(0.00-2.66)$ & 0.2025 \\
\hline \multicolumn{5}{|l|}{ Any prehospital defibrillation } \\
\hline No & 140 & 15 & Reference & \\
\hline Yes & 76 & 30 & $3.821(1.28-12.53)$ & 0.0196 \\
\hline
\end{tabular}

CI: Confidence interval; CPC: Cerebral performance category; CPR: Cardiopulmonary resuscitation; ROSC: Return of spontaneous circulation

interest in further studies. This is because while guidelines are developed mainly from Caucasian populations, ${ }^{24}$ these need to be suitably applied in an Asian ${ }^{7}$ or local context.

We identified predictors of mortality and good CPC/ OPC scores in our population. Modifiable factors identified include time between arrest and ROSC, diabetes, hypertension, initial CPR performed by EMS/private ambulance, prehospital defibrillation and $\mathrm{PCI} /$ placement of stent. Better education of the public can reduce the incidence and severity of chronic diseases such as diabetes and hypertension. As prehospital defibrillation and CPR performed by EMS/private ambulance are of significance in reducing mortality, possible measures to increase these may be to educate lay people on where to find and use automated external defibrillators and to regularly ensure our paramedics are up-to-date with their CPR skills. ${ }^{25}$ These findings as a whole strongly support a focus on basic life support measures through community and prehospital intervention to increase prompt and effective CPR and automated external defibrillator use, which have far greater effect on outcomes. ${ }^{25-27}$

Although there was some suggestion that TTM improved mortality and CPC in our study population, there was insufficient power to demonstrate statistical significance. We postulate that TTM may not have been complete in many patients and complications may not have been accounted for. The next step in this study will be to analyse specific TTM factors such as duration of TTM, temperature used, presence of overcooling and complications from TTM which can theoretically cause TTM to be less effective than it should have been. This is especially so in light of developments in TTM, such as a randomised-controlled trial (RCT) in 2013 by Nielsen et al showing that there were similar favourable outcomes in patients who underwent regimens of TTM set to $33^{\circ} \mathrm{C}$ and $36^{\circ} \mathrm{C} .{ }^{28}$ Another example is also evidence that time to target temperature plays a role in outcomes. ${ }^{29}$ Precautions must be taken because TTM can be associated with complications such as sepsis and renal failure. ${ }^{30}$ Clinicians taking care of such patients need to be well versed in managing patients with medical complexity beyond the cardiac issue itself. This parallels the trend of subspecialty training in cardiac intensive care. ${ }^{31}$ It can be postulated that benefits of TTM are realised in non-trial populations. As the finer technical details of TTM are investigated, ${ }^{32}$ clinician familiarity in treating TTM patients improves and more refined patient selection is achieved. As such, ongoing registries will continue to play an important role in informing care of OHCA patients. ${ }^{19,33}$ Having knowledge of these factors can thus aid us to utilise TTM to greatly benefit our patients.

Ethnicity has previously been reported to be a factor in affecting outcomes of patients undergoing TTM. A study by Jacobs et al in a single tertiary care hospital in the USA demonstrated that despite a strict TTM protocol, nonwhite patients were associated with poorer outcomes as compared to white patients, despite adjusting for possible confounders. ${ }^{34}$ This echoes a previous study performed by Agarwal et al which demonstrated that Hispanic ethnicity was associated with a poorer 1-year neurological outcome..$^{35}$ Chen et al studied an in-hospital cardiac arrest population and found that black survivors had a lower absolute rate of long-term survival after discharge from hospital, although 
they could not rule out effects of postdischarge care ${ }^{16}$ Ethnic differences in our local context have been shown to affect disease outcomes, such as in diabetes control. ${ }^{17}$ Our study consisted of a multiethnic Asian population. Based on the above studies, we would possibly have expected a difference in neurological outcomes. In our study (with Chinese as the reference group), Malays were less likely to have a good neurological outcome on multivariable analysis (adjusted OR $0.10,95 \% \mathrm{CI} 0.01-0.65, P=0.0319$ ) although this was not observed in Indians and other ethnicities. This finding deserves further investigation in future studies to understand whether it is an ethnic-specific or systems-specific factor that is resulting in this observed disparity.

A final discussion point would be that the protocols used in our study - in comparison to the 2 TTM trials mentioned-were different. In our study, TTM was initiated in the emergency department via external cooling and then initiation of internal cooling within 30 minutes. In the trial by the Hypothermia after Cardiac Arrest Study Group, temperature management was performed via an external cooling blanket ${ }^{3}$ and temperature was maintained between $32-34^{\circ} \mathrm{C}$ for 24 hours before rewarming. In the study by Bernard et al, cooling was initiated prehospital by paramedics via applying cold packs externally and continued in the emergency department. ${ }^{4}$ The total cooling period was 12 hours at $33^{\circ} \mathrm{C}$ in this study and thereafter they were rewarmed. In our study, our patients did not have prehospital cooling and instead of using external cooling alone, internal cooling was also performed. The target temperature in our study was also $34^{\circ} \mathrm{C}$. As there was heterogeneity in the initiation of cooling, the methods used to cool, the target temperature, the duration of cooling and the study population characteristics, it is therefore not surprising that we observed a non-significant neurological benefit although there was a trend towards this. Our paramedics are currently not trained to initiate TTM in the field, although this could be for further discussion with the relevant authorities to improve prehospital care. ${ }^{36,37}$ We believe that further RCTs, with a standardised protocol, would highlight further the external validity of the trials in different populations and health systems.

\section{Strengths and Limitations}

To the best of our knowledge, this study is the largest reported population of OHCA patients in a multiethnic Asian population. We were able to collect mortality and neurological outcome data over a 6-year period with a high percentage of data available for analysis $(99.6 \%$, 261/262). However, we do acknowledge that this was a single-centre study that may limit generalisation. As this was a retrospective study, and because PAROS did not capture the reasons why patients were not included for TTM, we cannot conclusively describe why certain patients who met the inclusion criteria were not started on TTM. We did not have information on time of TTM induction, duration of TTM, target temperature achieved, cooling methods for each group or complications experienced. Also, we could only demonstrate association and not causation in this observational study. Multicentre collaborative efforts to study TTM and well designed RCTs can be the focus of future efforts.

\section{Conclusion}

Adoption and outcomes of TTM postresuscitation varies and there is still a need to optimise management of the identified predictors of survival and good neurological outcomes while TTM is being used. Further longitudinal studies on TTM use in Asian populations are warranted.

\begin{abstract}
Acknowledgements
The authors would like to thank the contributions of physicians and nurses from the following institutions: Department of Emergency Medicine, Singapore General Hospital; Coronary Care Unit, Department of Cardiology, National Heart Centre, Singapore; and Intensive Care Unit, Department of Respiratory and Critical Care Medicine, Singapore General Hospital. This study was supported by grants from National Medical Research Council, Clinician Scientist Awards, Singapore (NMRC/CSA/024/2010 and NMRC/ CSA/0049/2013), Ministry of Health, Health Services Research Grant, Singapore (HSRG/0021/2012).
\end{abstract}

\section{Financial Disclosures}

Andrew FH Ho was supported by Khoo Clinical Scholars Programme, Khoo Pilot Award (KP/2019/0034), Duke-NUS Medical School and National Medical Research Council (NMRC/CS Seedfd/012/2018). Ching Hui Sia was supported by the Singapore Population Health Improvement Centre (SPHERiC) Fellowship.

\section{Conflict of Interest}

Marcus EH Ong has a licensing agreement and patent filing (Application no: 13/047,348) with Zoll Medical Corporation for a study titled 'Method of predicting acute cardiopulmonary events and survivability of a patient'. He is also a Scientific Advisor for Global Healthcare SG, a commercial entity that manufactures cooling devices.

\section{REFERENCES}

1. Chua MT, Chan GW, Kuan WS. Reversible causes in cardiovascular collapse at the emergency department using ultrasonography (REVIVEUS). Ann Acad Med Singapore 2017;46:310-6.

2. Oh YZ, Lee CT, Lim AT, Tong KL. Sports-related sudden cardiac deaths in Singapore - an eleven-year review. Ann Acad Med Singapore 2019;48:156-60

3. Hypothermia after Cardiac Arrest Study Group. Mild therapeutic hypothermia to improve the neurologic outcome after cardiac arrest. N Engl J Med 2002;346:549-56. 
4. Bernard SA, Gray TW, Buist MD, Jones BM, Silvester W, Gutteridge G, et al. Treatment of comatose survivors of out-of-hospital cardiac arrest with induced hypothermia. N Engl J Med 2002;346:557-63.

5. Nolan JP, Morley PT, Vanden Hoek TL, Hickey RW, Kloeck WG, Billi J, et al. Therapeutic hypothermia after cardiac arrest: an advisory statement by the advanced life support task force of the International Liaison Committee on Resuscitation. Circulation 2003;108:118-21.

6. Donnino MW, Andersen LW, Berg KM, Reynolds JC, Nolan JP, Morley PT, et al. Temperature management after cardiac arrest. Circulation 2015;132:2448-56.

7. Aibiki M, Chiang MC, Muengtaweepongsa S, Pothiawala S, Huang CH. Asian Targeted Temperature Management Task Panel Report. Ther Hypothermia Temp Manag 2017;7:16-23.

8. Ong ME, Shin SD, De Souza NN, Tanaka H, Nishiuchi T, Song KJ, et al. Outcomes for out-of-hospital cardiac arrests across 7 countries in Asia: the Pan Asian Resuscitation Outcomes Study (PAROS). Resuscitation 2015;96:100-8.

9. Tan TXZ, Hao Y, Ho AFW, Shahidah N, Yap S, Ng YY, et al. Inter-hospital variations in resuscitation processes and outcomes of out-of-hospital cardiac arrests in Singapore. J Emerg Crit Care Med 2019;3:21.

10. Lee BK, Park KN, Kang GH, Kim KH, Kim G, Kim WY, et al. Outcome and current status of therapeutic hypothermia after out-of-hospital cardiac arrest in Korea using data from the Korea Hypothermia Network registry. Clin Exp Emerg Med 2014;1:19-27.

11. Yokoyama H, Nagao K, Hase M, Tahara Y, Hazui H, Arimoto H, et al. Impact of therapeutic hypothermia in the treatment of patients with out-of-hospital cardiac arrest from the J-PULSE-HYPO study registry. Circ J 2011;75:1063-70.

12. Vattanavanit V, Bhurayanontachai R. Clinical outcomes of 3-year experience of targeted temperature management in patients with outof-hospital cardiac arrest at Songklanagarind Hospital in Southern Thailand: an analysis of the MICU-TTM registry. Open Access Emerg Med 2016;8:67-72.

13. Srivilaithon W, Muengtaweepongsa S. The outcomes of targeted temperature management after cardiac arrest at emergency department: a real-world experience in a developing country. Ther Hypothermia Temp Manag 2017;7:24-9.

14. Hung SW, Chen CC, Shih HC, Huang CF, Chen KC, Chong CF, et al. Are new resuscitation guidelines better? Experience of an Asian metropolitan hospital. Ann Acad Med Singapore 2010;39:569-75.

15. Ng M, Wong ASL, Chew HC, Shahidah N, Pek PP, Poh J, et al. Pilot prospective study of therapeutic hypothermia for treatment of post-cardiac arrest patients. Int J Cardiol 2014;173:612-3.

16. Chen LM, Nallamothu BK, Spertus JA, Tang Y, Chan PS. Racial differences in long-term outcomes among older survivors of in-hospital cardiac arrest. Circulation 2018;138:1643-50.

17. Hong CY, Chia KS, Hughes K, Ling SL. Ethnic differences among Chinese, Malay and Indian patients with type 2 diabetes mellitus in Singapore. Singapore Med J 2004;45:154-60.

18. Mok YH, Loke AP, Loh TF, Lee JH. Characteristics and risk factors for mortality in paediatric in-hospital cardiac events in Singapore: retrospective single centre experience. Ann Acad Med Singapore 2016;45:534-41.

19. Ong ME, Shin SD, Tanaka H, Ma MH, Khruekarnchana P, Hisamuddin N, et al. Pan-Asian Resuscitation Outcomes Study (PAROS): rationale, methodology, and implementation. Acad Emerg Med 2011;18:890-7.

20. Safar P. Cerebral resuscitation after cardiac arrest: summaries and suggestions. Am J Emerg Med 1983;1:198-214.

21. Yap LG, Shahidah N, Pothiawala S, Tan KBK, Wong ASL, Sewa DW, et al. Novel wearable cooling device for early initiation of targeted temperature management in the emergency department. J Emerg Crit Care Med 2020. Available at: http://jeccm. amegroups.com/article/view/5598. Accessed on 14 February 2020.

22. Harris PA, Taylor R, Thielke R, Payne J, Gonzalez N, Conde JG. Research electronic data capture (REDCap) - a metadata-driven methodology and workflow process for providing translational research informatics support. J Biomed Inform 2009;42:377-81.

23. Mader TJ, Nathanson BH, Soares WE $3^{\text {rd }}$, Coute RA, McNally BF. Comparative effectiveness of therapeutic hypothermia after out-ofhospital cardiac arrest: insight from a large data registry. Ther Hypothermia Temp Manag 2014;4:21-31.

24. Callaway CW, Donnino MW, Fink EL, Geocadin RG, Golan E, Kern KB, et al. Part 8: Post-Cardiac Arrest Care: 2015 American Heart Association Guidelines Update for Cardiopulmonary Resuscitation and Emergency Cardiovascular Care. Circulation 2015;132:S465-82.

25. Ho AFW, Hao Y, Pek PP, Shahidah N, Yap S, Ng YY, et al. Outcomes and modifiable resuscitative characteristics amongst pan-Asian out-of-hospital cardiac arrest occurring at night. Medicine (Baltimore) 2019;98:e14611.

26. Cummins RO, Ornato JP, Thies WH, Pepe PE. Improving survival from sudden cardiac arrest: the "chain of survival" concept. A statement for health professionals from the Advanced Cardiac Life Support Subcommittee and the Emergency Cardiac Care Committee, American Heart Association. Circulation 1991;83:1832-47.

27. Tanaka H, Ong MEH, Siddiqui FJ, Ma MHM, Kaneko H, Lee KW, et al. Modifiable factors associated with survival after out-of-hospital cardiac arrest in the Pan-Asian Resuscitation Outcomes Study. Ann Emerg Med 2018;71:608-17.e15.

28. Nielsen N, Wetterslev J, Cronberg T, Erlinge D, Gasche Y, Hassager C, et al. Targeted temperature management at $33^{\circ} \mathrm{C}$ versus $36^{\circ} \mathrm{C}$ after cardiac arrest. N Engl J Med 2013;369:2197-206.

29. Haugk M, Testori C, Sterz F, Uranitsch M, Holzer M, Behringer W, et al. Relationship between time to target temperature and outcome in patients treated with therapeutic hypothermia after cardiac arrest. Crit Care 2011;15:R101.

30. Soleimanpour H, Rahmani F, Golzari SE, Safari S. Main complications of mild induced hypothermia after cardiac arrest: a review article. J Cardiovasc Thorac Res 2014;6:1-8.

31. Fuster V. The (r)evolution of the CICU. Better for the patient, better for education. J Am Coll Cardiol 2018;72:2269-71.

32. Look X, LiH, Ng M, Lim ETS, Pothiawala S, Tan KBK, etal. Randomized controlled trial of internal and external targeted temperature management methods in post-cardiac arrest patients. Am J Emerg Med 2018;36:66-72.

33. Tan AT, Emmanuel SC, Tan BY, Teo WS, Chua TS, Tan BH. Myocardial infarction in Singapore: a nationwide 10-year study of multiethnic differences in incidence and mortality. Ann Acad Med Singapore 2002;31:479-86.

34. Jacobs CS, Beers L, Park S, Scirica B, Henderson GV, Hsu L, et al. Racial and ethnic disparities in postcardiac arrest targeted temperature management outcomes. Crit Care Med 2020;48:56-63.

35. Agarwal S, Presciutti A, Roth W, Matthews E, Rodriguez A, Roh DJ, et al. Determinants of long-term neurological recovery patterns relative to hospital discharge among cardiac arrest survivors. Crit Care Med 2018;46:e141-50.

36. Doctor NE, Yap S, Gan HN, Leong BSH, Goh ES, Chia MYC, et al. Recognition and treatment of out-of-hospital cardiac arrests by nonemergency ambulance services in Singapore. Ann Acad Med Singapore $2013 ; 42: 445-50$

37. Goh ES, Liang B, Fook-Chong S, Shahidah N, Soon SS, Yap S, et al. Effect of location of out-of-hospital cardiac arrest on survival outcomes. Ann Acad Med Singapore 2013;42:437-44. 


\section{Appendix}

\section{TTM Protocol}

\section{Induction Phase:}

- The Emergency Department will initiate cooling with cold saline infusion 2 L over 2 hours and ice packs on patient's axilla and groin until the cooling device is started in the CCU/MICU.

- Reach target temperature rapidly.

- Insert a temperature sensing foley catheter or rectal temperature probe or oesophageal temperature probe to monitor core temp.

- Record temp every 30 min until rewarming is complete (temperature data should be downloaded if possible or at least every 15 minutes during induction phase).

- Obtain a Glasgow Coma Score prior to giving paralytics.

- Sedate and paralyse to avoid shivering.

- Time to reach target temperature.

- The lowest temperature ever reached.

Sedation Protocol:

- IV Midazolam initial dose $0.02-0.08 \mathrm{mg} / \mathrm{kg}$ over $1-2 \mathrm{~min}$, then $0.04 \mathrm{mg} / \mathrm{kg} / \mathrm{hr}$ titrate up to 0.2 $\mathrm{mg} / \mathrm{kg} / \mathrm{hr}$ to achieve sedation, and if blood pressure tolerates.

Paralytic Protocol:

- Atracurium 0.4-0.5 mg/kg I.V. bolus then $5-10 \mathrm{mg} / \mathrm{kg} / \mathrm{min}$ and titrate as needed

- CCU/MICU to place patient on Heat, Moisture, Exchange (HME) unit during cooling rather than heated wire circuit (ventilator).

- Initiate cooling device according to manufacturers' protocol.

- Set the target temperature on the cooling unit to $34^{\circ} \mathrm{C}$.

- Ice packs to axilla and groin can be removed at this point.

- Once patient temperature of $34^{\circ} \mathrm{C}$ is achieved (will take about 3-8 hrs), keep target temperature at $34^{\circ} \mathrm{C}$.

\section{Maintenance Phase:}

- Target MAP >80 mmHg to maintain cerebral perfusion.

- Administer vasopressors through central venous access; as per physician's discretion.

- If hypertensive, short acting vasodilators such as Nitroglycerine may be used.

- Keep CVP 10-15 mmHg or PCOP >12 mmHg using Normal Saline or Lactated Ringers (CVP or PCOP monitoring not mandatory) per doctor's order.

- Use Low Molecular Weight heparin (LMWH), Enoxaparine at a dose of $40 \mathrm{mg}$ once daily, if no contraindications.

- Lacrilube to both eyes quarter hour while receiving paralytics.

- Skin care to avoid cold-related injury. 
- Frequent suctioning and pulmonary toilet.

- Nursing interventions to avoid complications of immobility (DVT, UTI, skin breakdown).

- Follow mixed venous saturation (Sv02) when available per doctor's orders rather than cardiac index since the $\mathrm{O}^{2}$ extraction ratio will be very low due to a markedly decreased metabolic demand from hypothermia and paralytics. Avoid dobutamine or other arrhythmogenic inotropes if $\mathrm{Sv} 0^{2}$ is close to $70 \%$.

- Stress Ulcer Prophylaxis: Famotidine 20 mg IV q 12 hr, or Famotidine 20 mg NGT q 12 hr per doctor's order.

- EEG monitoring is recommended to detect seizures in the paralysed patient.

- Manage status epilepticus with antiepileptic medication according to standard procedure.

- Any cardiac arrhythmias should be treated with standard anti-arrhythmia protocols.

- Potential laboratory abnormalities associated with hypothermia:

\section{Potential Lab Abnormalities Treatment}

Increased amylase $\rightarrow$ No intervention unless persistent after rewarming. Increased LFT's $\rightarrow$ No intervention unless persistent after rewarming.

Increased serum glucose $\rightarrow$ Follow insulin protocol.

Decreased $\mathrm{K}+, \mathrm{Mg}$, Phosp, $\mathrm{Ca} \rightarrow$ Correct as needed.

Increased lactate $\rightarrow$ Optimise oxygen delivery.

Metabolic acidosis $\rightarrow$ Optimise oxygen delivery.

Thrombocytopaenia $\rightarrow$ Correct if $<30 \mathrm{k}$, or to $>50 \mathrm{k}$ if active bleeding.

Leukopaenia $\rightarrow$ No intervention unless persistent after rewarming.

- If the patient needs to go for cardiac catheterisation, the cooling device should accompany the patient. The patient may be unplugged from the device enroute, but cooling should be reinstituted on arrival at the catheterisation lab.

- In the event of cardiac arrest requiring defibrillation or cardioversion, it is suggested that the lower energy setting be initially employed, with escalating energy settings as necessary.

\section{Rewarming Phase:}

- After 24 hours at $34^{\circ} \mathrm{C}$, rewarm passively to $36.5^{\circ} \mathrm{C}$ by setting the cooling unit accordingly. Aim to increase temperature by $1^{\circ} \mathrm{C}$ every 4 hours, and achieve rewarming over at least $12 \mathrm{~h}$. Warm no faster than $1^{\circ} \mathrm{C}$ in 4 hours.

- If experiencing difficulty rewarming as above, use heated ventilator air to provide core rewarming.

- Discontinue all potassium in IVF during rewarming per doctor's order. Cases of severe hyperkalemia have been reported on rewarming. Represents a transcellular shift rather than true $\mathrm{K}+$ depletion.

Documentation

- Temperature every 1 hour or more frequently.

- V/S every 1 hour or more frequently.

- Glasgow Coma scores on initiation and every 4 hours.

- Skin evaluation and care given every 2 hours or more frequent.

- Time of initiation and completion of cooling and reaching target temperature.

- Patient's response to procedure.

- All completed data will then be collected and sent to the research team for data management.

- At the end of rewarming, after target warm temperature achieved, to inform trial coordinator to perform data download from the machine data $\log$.

- Temperature variation.

- Other cooling or warming methods used to reach and/or maintain target. 